\section{Congenital Complete Absence of Pericardium Presenting with Chest Pain: MRI Characteristics}

Madhan Nellaiyappan1, Hany Demo ${ }^{2}$,

Hesham R. Omar ${ }^{3}$, Tony Fattouch 4 , Orestis V Pappas ${ }^{1}$, Devanand Mangar ${ }^{5}$, Enrico M. Camporesi ${ }^{6}$

\section{Abstract}

Congenital absence of the pericardium is a very rare anomaly which has an incidence of 1 in 14000 cases in autopsy studies. Defects in the pericardium are either partial or complete with complete defects having a much benign course than partial defects. Partial defects are associated with complications like strangulation of a herniated cardiac chamber or coronary artery stenosis from external compression. Absence of the left pericardium is the most frequently encountered type of defect which accounts for 2/3 of the cases. We herein report a case of complete absence of the entire pericardium which is an extremely rare subtype with associated Cardiac magnetic resonance images.

\section{Keywords}

Congenital Absence of Pericardium; Complete Absence of Pericardium; Magnetic Resonance Imaging.

\section{Introduction}

Congenital absence of the pericardium is a very rare anomaly which is usually asymptomatic. But it can also be symptomatic and infrequently associated with life threatening complications like strangulation of heart chambers and sudden cardiac death making it a clinically relevant entity. Congenital absence of the pericardium is usually an autopsy diagnosis or an incidental finding at the time of cardiothoracic surgeries $[1,2]$. Its incidence in autopsy studies was reported as 1 in 14000 [3]. In a population of cardiovascular surgical patients, its incidence was 1 in 2267 patients [1]. Defects can either be partial or complete. The most common type is a left sided pericardial absence which accounts for 2/3 of all congenital pericardial defects [4]. Complete absence of the entire pericardium (left and right sided) is consi-
1 Internal Medicine Department, Allegheny General Hospital, Pittsburgh, Pennsylvania, USA.

2 Cardiology Department, Advocate Illinois Masonic Medical Center, Chicago, Illinois, USA.

3 Internal Medicine Department, Mercy Medical Center, Clinton, Iowa, USA.

4 Cardiothoracic imaging department University of South Florida, Tampa, Florida, USA

5 Chief of Anesthesia, Tampa General Hospital; CEO, FGTBA and Regional Medical Director, TEAMHealth, Tampa, Florida, USA.

6 Professor of Surgery/Anesthesiology, Professor of Molecular Pharmacology and Physiology, University of South Florida, FGTBA and TEAMHealth, Tampa, Florida, USA.

\section{Contact information:}

\section{Madhan Nellaiyappan.}

Address: Internal Medicine Department Allegheny Health Network,Pittsburgh, Pennsylvania, USA.

ほ drnmadhan@gmail.com 
dered very rare and accounts for only a fraction of the cases reported. We present a case of congenital complete absence of the pericardium and describe the characteristic cardiac magnetic resonance imaging (MRI) findings.

\section{Case report}

A healthy 48-year-old male presented for outpatient exercise echocardiography stress test. He had been complaining of positional pleuritic chest pain. His past medical history and family history were unremarkable. He was on no medications and denied illicit drug use, tobacco or alcohol abuse. Physical examination revealed a blood pressure of 114/68 $\mathrm{mmHg}$ and a pulse of 54 beat/minute. There was no jugular venous distension and lungs were clear to auscultation. There was mild pectus excavatum.
Cardiac examination was remarkable for lateral displacement of the apex to the mid-axillary line, with a grade II/VI systolic murmur heard best at the lower left sternal border with no radiation. A 12-lead electrocardiogram (ECG) showed sinus bradycardia with heart rate of 52 beats per minute, incomplete right bundle branch block and poor $\mathrm{R}$ wave progression in precordial leads. Cardiac stress test was negative for inducible ischemia. Echocardiogram showed various abnormalities including unusual echocardiographic windows, cardiac hypermobility, abnormal swinging motion and abnormal ventricular septal motion. Based on the abnormal echocardiogram findings, a Cardiac MRI was obtained which confirmed the diagnosis of congenital absence of the entire pericardium (Figure 1).

Figure 1: MRI images of the patient with congenital complete pericardial absence is illustrated in panels $A, B$ and $C$. MRI images in panels $D, E, F$ belong to a patient with no pericardial absence and have been provided for comparison.
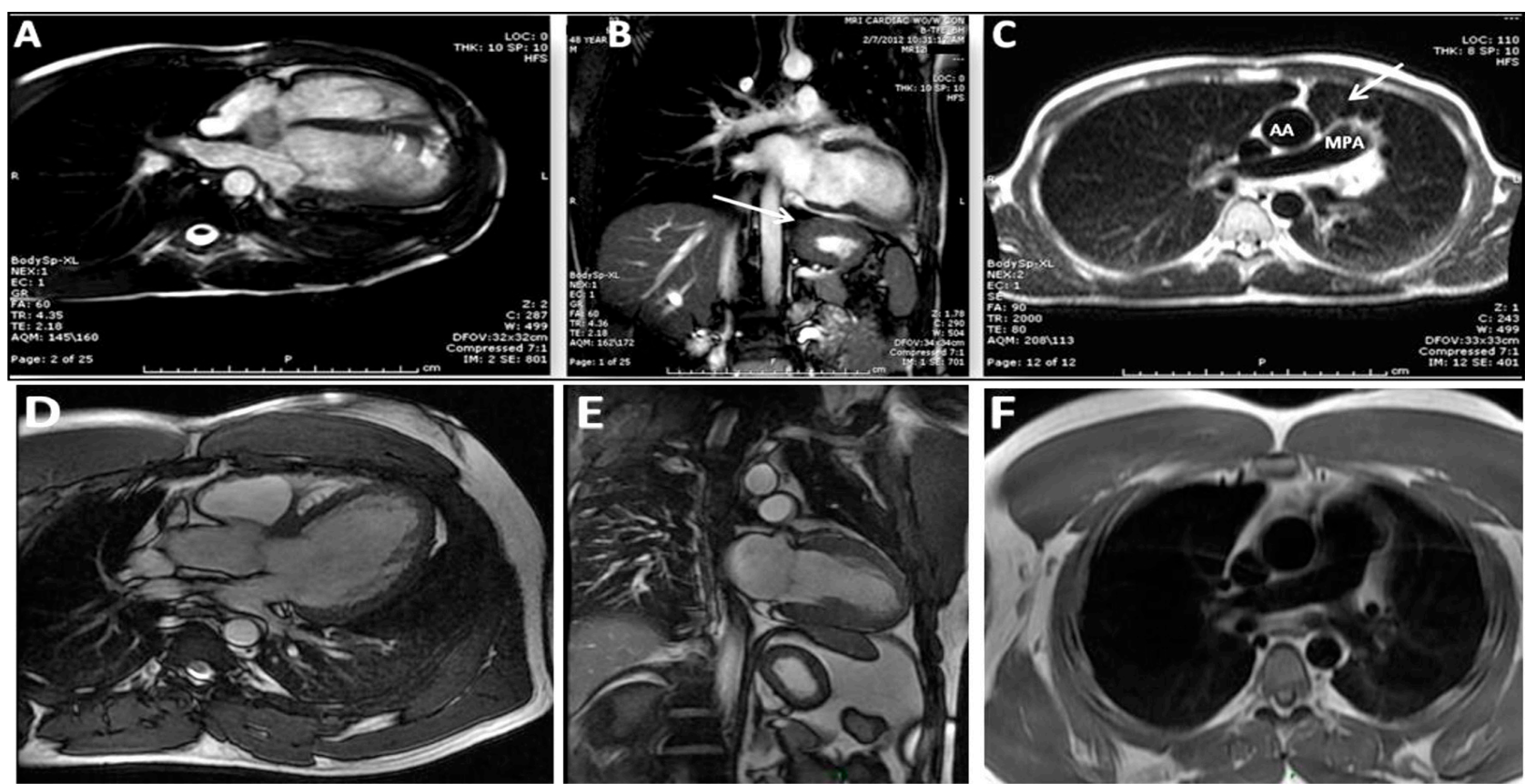

Panels A and B: Balanced Turbo Field Echo MRI demonstrates non visualization of the entire pericardium, levoposition of the heart with the apex towards the mid axillary line (A), and lung tissue interposition between the inferior surface of the heart and the diaphragm (B) which confirms the absence of pericardium. Panel C: T2 weighted Turbo Spin Echo MRI shows a "tongue" of lung which interposes between the main pulmonary artery and the aorta. Panel D: The apex of the heart is directed towards to the midclavicular line (D). There is no lung tissue between the inferior surface of the heart and diaphragm (E). Panel (F) demonstrates no lung tissue interpositioning between the aorta and the pulmonary artery. Also note the subtle mediastinal shift of the great vessels in panel (C) when compared to panel $(F)$ which would also indicate the levoposition of the heart. 


\section{Discussion}

Congenital defects of the pericardium are thought to result from inappropriate, early atrophy of the ducts of Cuvier which supply blood to the developing pleuropericardial membranes [3]. In most instances, these atrophies are left-sided [3]. Likewise, congenital absence of pericardium is frequently left sided. They have been associated with intracardiac anomalies like atrial septal defect, ventricular septal defect, tetralogy of Fallot [1] and extracardiac anomalies like diaphragmatic hernia [2], lung anomalies like left lung hypoplasia and bronchogenic cyst [5]. The presented case herein exemplifies an isolated complete absence of the entire pericardium. Analysis of published data on complete absence of the entire pericardium reveals that though the defect has been present from birth, the diagnosis has been made across different age groups, from as early as the neonatal age group to as late as the eighth decade of life $[2,6]$. Almost always, neonatal and infantile patients are being diagnosed at the time of surgery for other congenital anomalies usually a congenital diaphragmatic hernia [2]. Reported presenting features of complete pericardial absence in adults include atypical chest pain of varying severity, palpitations, shortness of breath or incidental discovery at the time of cardiothoracic surgeries [4]. Due to vague and atypical symptoms, congenital absence of pericardium can mimic other conditions such as acute coronary syndromes, valvular heart disease like mitral prolapse, pulmonic stenosis, tumours of the lung or heart. Chest pain is thought to be secondary to tension from pleuropericardial adhesions, lack of pericardial cushioning, and undue torsion or strain on the great vessels as they serve as the only anchor for the heart. [7]

Defects in the pericardium can be complete or partial. Complete absence of the pericardium usually carries an excellent prognosis and has only been rarely associated with traumatic transection of aorta, type A aortic dissection, sudden death due to possible torsion/trauma to great vessels owing to cardiac hypermobility $[1,6,8]$. Partial defects have been much more frequently associated with life threatening complications that include herniation and strangulation of a heart chamber or stenosis of a coronary artery leading to dysrhythmias, angina and sudden cardiac death $[1,9]$.

The diagnosis of congenital pericardial absence though rare has been associated with pathognomonic and tell-tale signs on imaging. Chest radiographs in patients without other obscuring congenital anomalies reveal levoposition of the heart, irregular left heart border, prominence of main pulmonary artery (MPA), and lung tissue interposition between the MPA and aorta (which are usually enclosed together by pericardium without lung tissue in-between) and also between the heart and diaphragm [10]. On transthoracic echocardiogram, unusual echocardiographic windows, cardiac hypermobility, abnormal ventricular septal motion and abnormal swinging motion of the heart are frequently encountered [5].

Magnetic resonance imaging is a noninvasive imaging modality which can definitively diagnose absence of pericardium and is considered the gold standard for evaluation of the pericardium. [7] Abnormal orientation of the heart where it is displaced to the left and posterior aspect, previously described as levoposition; is noted with congenital complete pericardial absence. MRI will also reveal excessive myocardial mobility and interposition of lung tissue between aorta and MPA. Non visualization of pericardium on MRI should not be utilized as the sole evidence to diagnose congenital absence of pericardium as the low signal intensity from the adjacent lungs and paucity of epicardial fat over the left atrial appendage and left ventricle can interfere with reliably identifying the pericardium [11]. Diagnosis should not be made without associated levoposition of the heart as in our case, or regional bulging of the heart chamber in partial defects [11].

Complete absence or small defects seldom require any treatment [10] and if needed may be li- 
mited to activity modification. Surgical procedures are usually done in patients with partial defects with associated or impending herniation of a cardiac chamber or at the time of other cardiovascular procedures [1]. Partial defects are either closed or enlarged depending on size to prevent herniation of vital structures [1]. Surgical reconstructions of the pericardium in patients with debilitating symptoms from left sided absence or complete pericardial absence have also been reported [10]. Surgery -which is not the norm- would aim for stabilization of the heart by using prosthesis/peritoneum to recreate a pericardial covering $[6,10]$.

\section{Conflict of interest}

There are no conflicts of interest to disclose

\section{Abbreviations}

AA: ascending aorta; MPA: main pulmonary artery.

\section{References}

1. Van Son J A, Danielson GK., Schaff H V., Mullany C J, Julsrud $P R$, Breen J F. Congenital Partial and Complete Absence of the Pericardium. Mayo Clinic proceedings. 68. 8. August 1993.7437

2. Tebruegge M O, Rennie J M, Haugen S E. Congenital absence of the pericardium associated with congenital diaphragmatic hernia and hepatic hemangioendothelioma: case report and review of the literature. Pediatr Surg Int (2005) 21: 557-559

3. Green D W, Meyers B F, Bhalla S, Hall B L Complete Absence of the Pericardium in a Patient With Multiple Injuries, J Trauma. 2007; 63:E66 -E68.

4. Abbas AE, Appleton CP, Liu PT, Sweeney JP. Congenital absence of the pericardium: case presentation and review of literature. Int J Cardiol. 2005 Jan; 98(1):21-5.

5. Ozpolat B, Doğan OV. Absence of pericardium in combination with bronchogenic cyst, atrial septal defect, mitral valve prolapsus and hypospadias. Anadolu Kardiyol Derg. 2009 Oct 9; 9(5):E16-7.

6. Meunier JP, Lopez S, Teboul J, Jourdan J. Total pericardial defect: risk factor for traumatic aortic type A dissection. Ann Thorac Surg. 2002 Jul; 74(1):266
7. Shah $A B$, Kronzon I. Congenital defects of the pericardium: a review. Eur Heart J Cardiovasc Imaging. 2015 Aug; 16(8):821-7.

8. Ventura F, Neri M, De Stefano F, Fineschi V. Sudden cardiac death in a case of undiagnosed pericardial agenesis. Rev Esp Cardiol. 2010; 63: 1103-1105

9. Amiri A, Weber Ch, Schlosser V, Meinertz T H. Coronary Artery Disease in a Patient with a Congenital Pericardial Defect. Thorac cardiovasc Surg 1989; 37(6): 379-381

10. Gatzoulis M A, Munk M-D, Merchant N, Van Arsdell G S, McCrindle B W, Webb G D. Isolated Congenital Absence of the Pericardium: Clinical Presentation, Diagnosis, and Management Ann Thorac Surg 2000; 69:1209-15

11. Rajiah P. Cardiac MRI: Part 2, Pericardial Diseases. American Journal of Roentgenology October 2011, Volume 197, Number 4. http://www.ajronline.org/doi/abs/10.2214/AJR.10.7265
Publish in International Archives of Medicine

International Archives of Medicine is an open access journal publishing articles encompassing all aspects of medical science and clinical practice. IAM is considered a megajournal with independent sections on all areas of medicine. IAM is a really international journal with authors and board members from all around the world. The journal is widely indexed and classified Q1 in category Medicine. 